\title{
Developments in HF Propagation Predictions to Support Communications with Aircraft on Trans-polar Routes
}

\author{
E. M. Warrington ${ }^{1}$, N. C. Rogers ${ }^{2}$, A. J. Stocker ${ }^{1}$, D. R. Siddle ${ }^{1}$, H. A. H. Al-Behadili ${ }^{1}$, \\ F. Honary ${ }^{2}$, M. J. Beharrell ${ }^{2}$, D. H. Boteler ${ }^{3}$, D. W. Danskin ${ }^{3}$, and N. Y. Zaalov ${ }^{4}$ \\ ${ }^{1}$ Department of Engineering, University of Leicester, Leicester LE1 7RH, UK \\ ${ }^{2}$ Department of Physics, Lancaster University, Lancaster LA1 4YW, UK \\ ${ }^{3}$ Natural Resources Canada, Ottawa K1A 0Y3, Canada \\ ${ }^{4}$ St. Petersburg State University, Ulyanovskaya 1, Petrodvorets, St. Petersburg 198504, Russia
}

\begin{abstract}
Commercial airlines began operations over polar routes in 1999 with a small number of proving flights. By 2014 the number had increased to in excess of 12,000 flights per year, and further increases are expected. For safe operations, the aircraft have to be able to communicate with air traffic control centres at all times. This is achieved by VHF links whilst within range of the widespread network of ground stations, and by HF radio in remote areas such as the Polar regions, the North Atlantic and Pacific where VHF ground infrastructure does not exist. Furthermore, the Russian side of the pole only has HF capability. This has created a demand for improved HF nowcasting and forecasting procedures to support the polar operations, which are the subject of this paper.
\end{abstract}

\section{INTRODUCTION}

Communications within the high latitude region is of growing importance for civil airlines operating trans-polar routes (see Figure 1) as these may form the shortest path between significant destinations (e.g., New York to Hong Kong), reducing travel time, cost and carbon emissions. Operation over polar routes began with a small number of flights in 1999, and by 2014, polar routes were operated by more than 10 major airlines, with over 12,000 cross-polar flights. However, in the polar cap above $82^{\circ} \mathrm{N}$ geostationary satellites lie below the horizon, and geographic and geopolitical considerations mean there are limited VHF radio air-traffic control facilities. Thus HF radio propagation via the ionosphere is of critical importance in maintaining communications. Adverse space weather conditions, leading to ionospheric disruption that in turn affects HF radio propagation, is of critical importance when considering whether polar routing is viable in the hours in advance of a flight (forecasting) and to the management of HF communications during a flight (nowcasting). Our research is currently directed towards the nowcasting and forecasting requirements. There are two aspects: (a) absorption, and (b) ray path characteristics.

Space weather events can influence the ionosphere in a number of ways, and these can be particularly pronounced at high latitudes (i.e., within the auroral zone and polar cap). The most severe space weather events lead to a total loss of communications within the HF band (a radio blackout) via strongly enhanced D-region absorption. More commonly, events of intermediate severity can lead to disruption of communications that may be managed by appropriate frequency selection, relaying of messages, and possibly by spatial diversity if the operational configuration is such as to allow this.

In addition to absorption, it is also necessary to take into account the presence of various features that are prevalent in the high latitude regions (in particular patches, arcs, auroral zone irregularities and the mid-latitude trough) that significantly affect the propagation of the radio signals. Propagation coverage predictions make use of numerical ray tracing to estimate the ray paths through a model ionosphere. Initially, a background ionospheric model is produced, which is then perturbed to include the various ionospheric features. The results of the ray tracing predictions may then be combined with absorption predictions to provide an overall estimate of signal strength and coverage over the region of interest.

\section{PROPAGATION MODEL}

Extensive HF propagation measurements have been made by the University of Leicester and colleagues at northerly latitudes over a number of years [1-5]. Of particular relevance to this paper, 


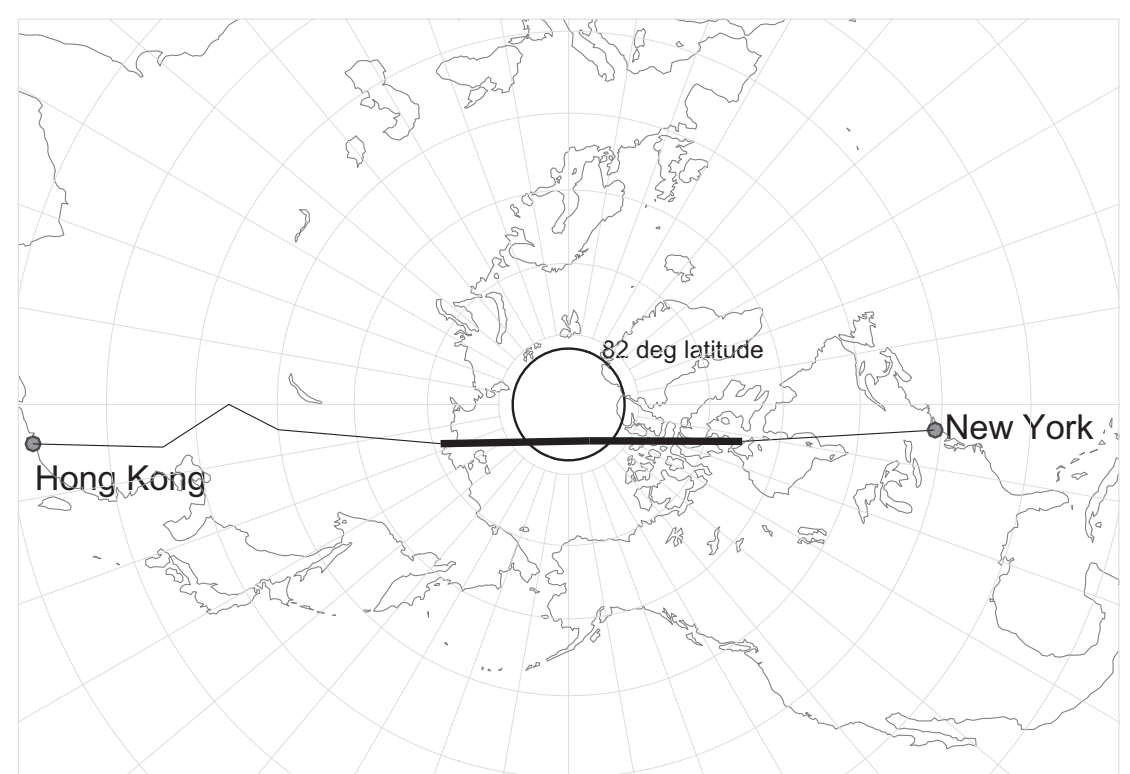

Figure 1: Example trans-polar route from New York to Hong Kong via Polar 3. The bold line indicates where communication is out of range of the VHF infrastructure and is only possible via HF links.

measurements undertaken in the polar cap found that the presence of convecting patches and sunaligned arcs of enhanced electron density can lead to signals arriving in directions displaced from the great circle path by up to $100^{\circ}[1,2]$. Patches are formed in the dayside auroral oval [6] during periods of southward directed Interplanetary Magnetic Field (IMF) $\left(B_{z}<0\right)$ and the associated high levels of geomagnetic activity and generally convect in an anti-sunward direction across the polar cap into the nightside auroral oval, whereas arcs occur when geomagnetic activity is low and the IMF is directed northward $\left(B_{z}>0\right)$ and drift in a duskwards direction [7]. We have undertaken measurements of signals received over a number of paths, including direction of arrival, time of flight (TOF) and signal strength with the specific aim of validating and developing our modelling procedures.

To estimate signal coverage, we use an ionospheric ray-tracing model [8] in conjunction with HF soundings, GPS Total Electron Content (TEC) measurements and other geophysical data as inputs to define a model ionosphere suitable for use with the ray tracing code. A realistic background ionosphere based on the observations is combined with localised ionospheric features (e.g., polar patches and arcs), D-region absorption and HF-transmitter/receiver antenna gain patterns in order to predict radio propagation behaviour. The area coverage expected from a transmitter at a given location is then estimated by ray-tracing through the modelled ionosphere. A large number of rays launched in an azimuth / elevation grid from the transmitter are traced through the model ionosphere. Each ray is assigned a power depending upon the transmitter power and antenna radiation pattern, absorption is added for each transit of the D-region taking into account the location of the transit, and the signal strength at the receiver estimated summing the ray power in the area around the receive antenna. Example coverage maps produced by this process for an $8.0 \mathrm{MHz}$ transmitter located at Qaanaaq, Greenland are presented in Figure 2. The effect of introducing patches into the model is particularly marked in this instance.

Our early work $[2,9]$ generated the background ionosphere based on ionosonde measurements made in the high latitude regions. As there are few ionosondes in the polar cap, an approach was adopted where measurements were extrapolated east and west of the ionosonde location based on the local time. We are currently developing an approach based around the IRI [10]. In order to generate a background ionosphere based on the IRI, but adjusted for real-time inputs, the following method has been adopted. Measurements of sTEC are taken from about 60 GPS stations located at mid and high latitudes. The IG and RZ solar activity indices are adjusted for each latitude and longitude until slant TEC (sTEC) values derived from the IRI match the observations. The other ionospheric parameters are then derived from the IRI. In order to test this approach, the foF2 values derived from the IRI driven by the slant TEC from 12 GPS stations are compared with the observed values of foF 2 from 12 nearby ionosondes. An example of the outcome of this process for 

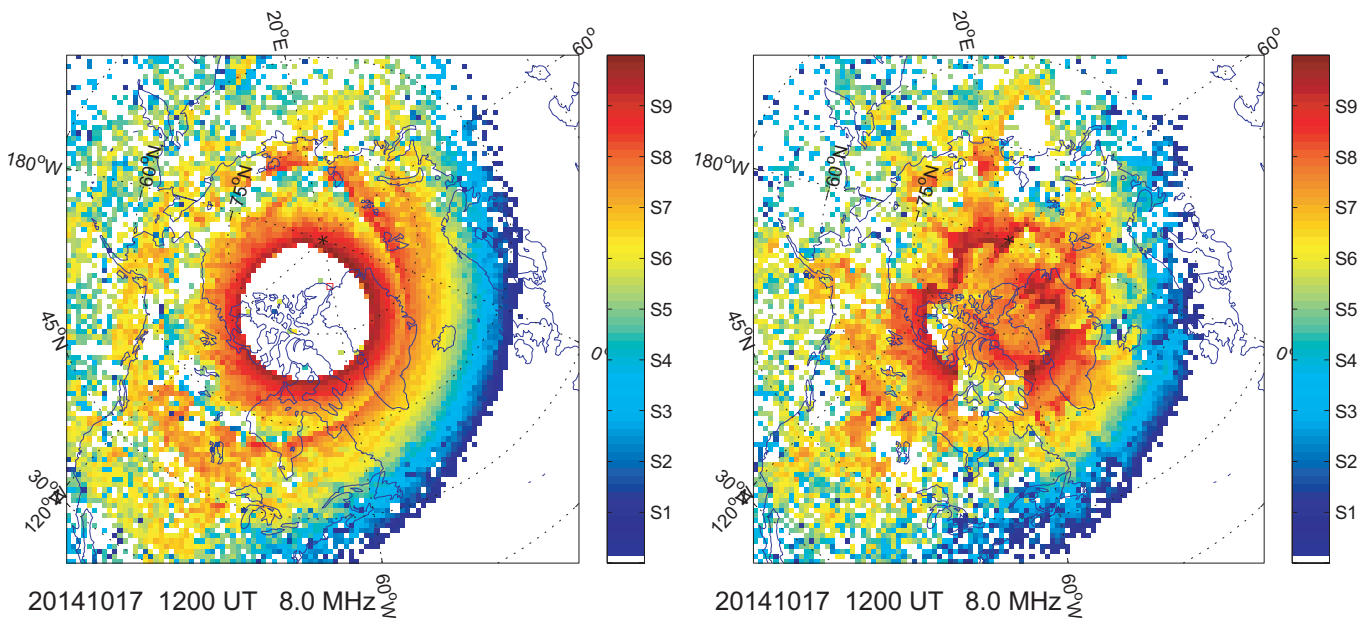

Figure 2: Coverage predicted for the Qaanaaq transmitter at $8.0 \mathrm{MHz}$ using the ray-tracing model for a $1 \mathrm{~kW}$ transmitter and monopole antennas at both transmitter and receiver. 12:00 UT, 17 October 2014. The left hand frame is for the background ionosphere, and the right hand frame has patches included in the simulation. Signal strength is indicated by the colour scalein S-units, the scale commonly used on HF communications receivers with $\mathrm{S} 1$ being very weak $(-121 \mathrm{dBm})$, stepping in $6 \mathrm{~dB}$ increments per S-unit to S9 $(-73 \mathrm{dBm})$ indicating a relatively strong signal, and then in $\mathrm{dB}$ exceeding $\mathrm{S} 9$.

a single mid-latitude station is presented in Figure 3.

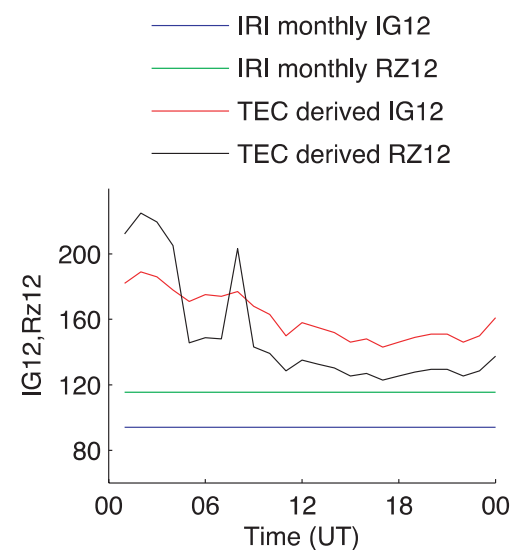

(a)

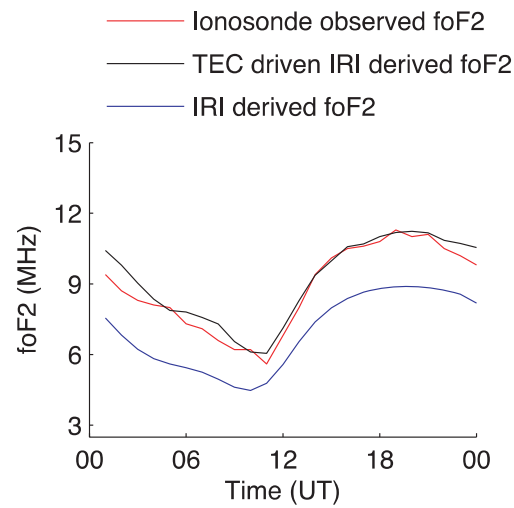

(b)

Figure 3: (a) The values of IG and RZ required to give good agreement between measured sTEC and IRIderived sTEC for the GPS receiver located at Bar Harbor, USA. (b) foF2 values obtained from the IRI with the sTEC-derived IG and RZ values compared with the foF2 values measured at nearby Millstone Hill, USA for 1 April 2014.

In the left hand panel, the IG and RZ values required to obtain agreement between the sTEC observed by GPS and that derived from the IRI are presented together with the standard values used in the IRI. For this example, the IG and RZ values are higher than the monthly values usually used by the IRI and vary with time. In the right hand panel, the foF 2 values derived from the IRI are lower than those observed by the ionosonde by a few MHz. Once TEC is used to drive the IRI, then the outcome is improved with a much closer agreement. The RMS errors between the observed foF 2 and the values derived from the IRI (standard and TEC driven) for 12 ionosonde stations indicate that the RMS error is usually less for the TEC driven IRI, i.e., the performance is improved, for all of the selected stations for this day.

\section{ABSORPTION MODEL}

The absorption model provides a map of D-region ionospheric absorption updated in real-time. Elements of the absorption model also allow for the inclusion of publicly available forecast parameters 
such as geomagnetic activity indices and solar energetic proton flux estimates, which may extend the predictions hours or days in advance.

HF radio absorption is largely due to enhanced electron density in the D-region ionosphere which alters the complex refractive index of the medium. The three principal ionising mechanisms are: (a) photoionisation from solar X-rays; (b) collisional ionisation from the enhanced energetic $(>1 \mathrm{MeV})$ proton flux associated with Solar Proton Events (SPEs); and (c) ionisation by energetic $(>20 \mathrm{keV})$ magnetospheric electrons precipitating into the auroral regions. The modelling of these three elements is discussed below.

Solar X-ray generated absorption is significant during M- and X-class solar flares and it causes short-lived absorption spikes (or short-wave fadeout (SWF)) on the dayside of the Earth. These are modelled using real-time $0.1-0.8 \mathrm{~nm}$ X-ray flux measurements from one of the NOAA Geostationary Operational Environmental Satellites (GOES), using the empirical relation of [11 (p.49)] which depends on solar-zenith angle, $\chi$, and radio frequency.

SPEs can dominate HF radio absorption across the polar cap region in the hours and days following large solar flares and interplanetary coronal mass ejections. During an SPE a high flux of energetic protons enter the Earth environment and causes up to several decibels of Polar Cap Absorption (PCA) at $30 \mathrm{MHz}$, with absorption at lower frequencies increasing as a power law with exponent of approximately 1.5 to 2 . At lower latitudes, most protons have insufficient rigidity (momentum per unit charge) to overcome geomagnetic forces and access the D region. The rigidity cutoff boundary latitude decreases with strengthening magnetospheric ring currents and has been modelled by Smart et al. [12] as a function of geomagnetic indices $K_{p}$ and $D_{s t}$. PCA is several times greater (in $\mathrm{dB}$ ) during the day than during the night, mainly due to higher effective electron recombination rates in the night-time D-region.

A widely used empirical model of PCA is the NOAA D-Region Absorption Prediction (DRAP) model [13]. This model relates the absorption, $A(\mathrm{~dB})$ to the flux of solar protons, $J$ (measured in real-time from a GOES satellite) above a certain energy threshold, $E_{t}(\mathrm{MeV})$, according to the relation, $A=m \sqrt{J\left(E>E_{t}\right)}$, where fixed parameters $m$ and $E_{t}$ are chosen from historical archive measurements, separately for day and night conditions.

The DRAP model framework has been developed into a new Polar Cap Absorption Model (PCAM) $[14,15]$ which assimilates absorption measurements from a network of riometers in the Global Riometer Array (GloRiA) (Figure 4), many of which provide real-time data online [16]. The parameters $m$ and $E_{t}$ - which may change from day to day depending on ionospheric composition and temperatures - are optimised using an age-weighted non-linear regression to the riometer

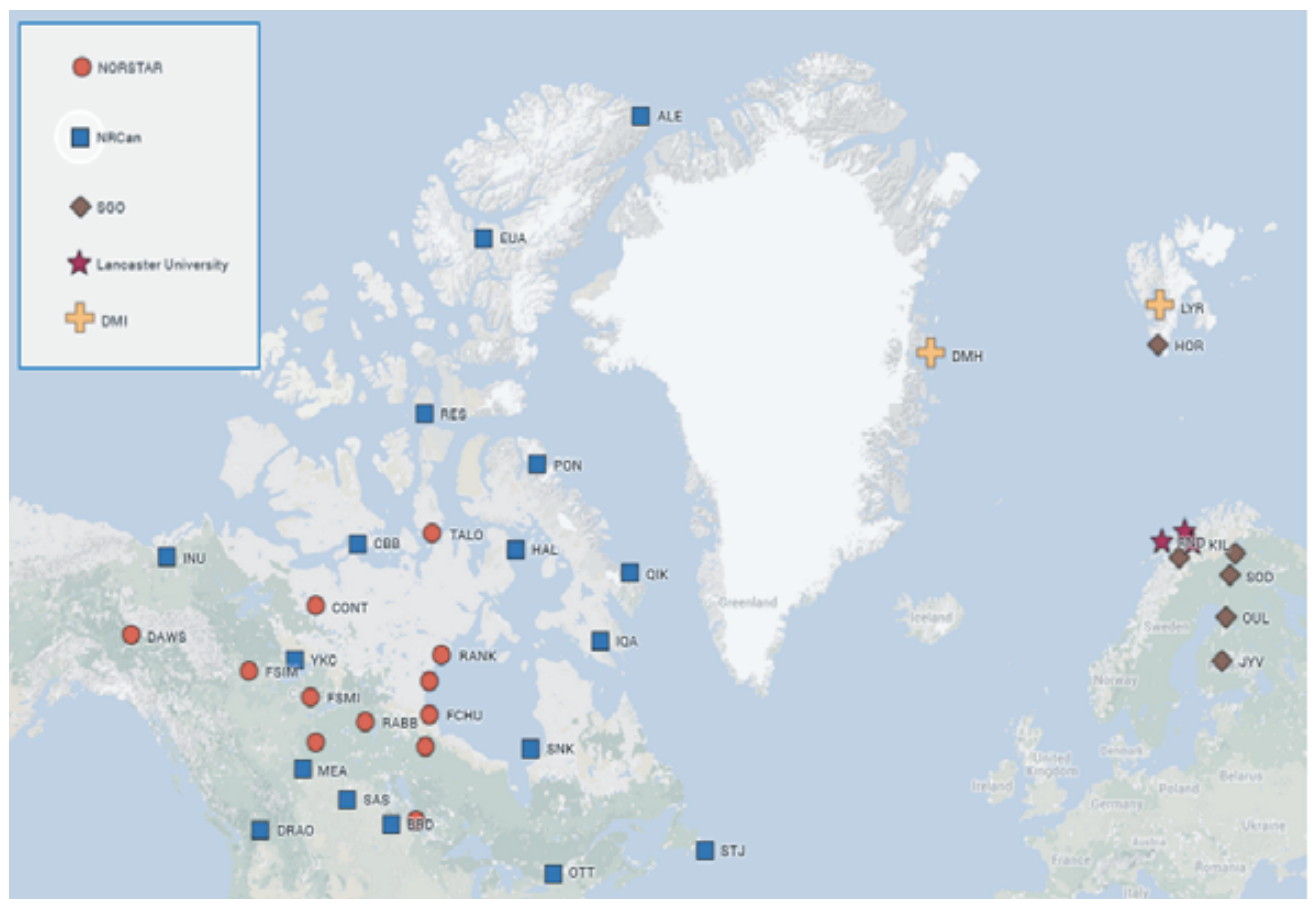

Figure 4: Selected riometers in the Global Riometer Array (GloRiA). 
measurements. These parameters vary rapidly at twilight and the transition zenith angles vary greatly from day to day [17]. Therefore the zenith angles of the twilight transition (fixed at 80 and $100^{\circ}$ in the DRAP model) are also optimised (independently at sunrise and sunset) by assimilation to recent riometer measurements. Figure 5 presents an example of a fitting such a model for sunset transitions during the SPE of April 2002 at the Ft Churchill riometer (labelled FCHU in Figure 5), illustrating the optimisation of $m$ for day and night (labelled $m_{d}$ and $m_{n}$ respectively), and the zenith angle limits of the twilight region $\chi_{l}$ and $\chi_{u}$. A further optimisation is applied to the latitude of the rigidity cutoff determined by Smart et al. [12].

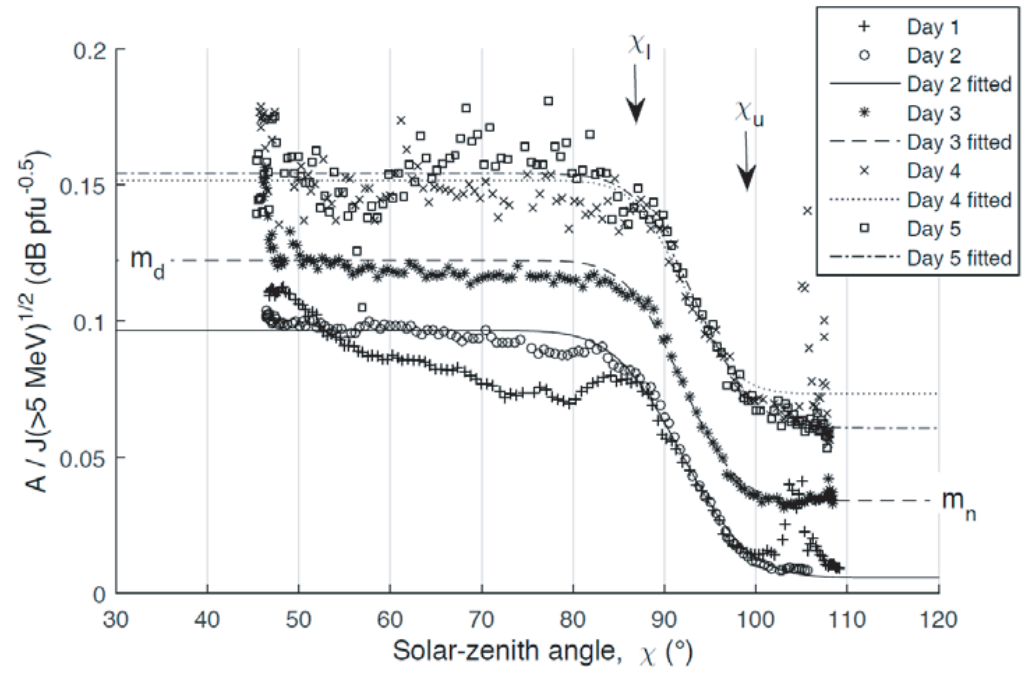

Figure 5: An example of fitting PCA model parameters $m$ (day and night), $\chi_{l}$ and $\chi_{u}$ to Ft Churchill riometer measurements (points) of $30 \mathrm{MHz}$ absorption and GOES measurements of proton flux. Model fits (lines) are optimised for sunset periods for 21-25 April 2002 (the plots cover the 12-24 local time period).

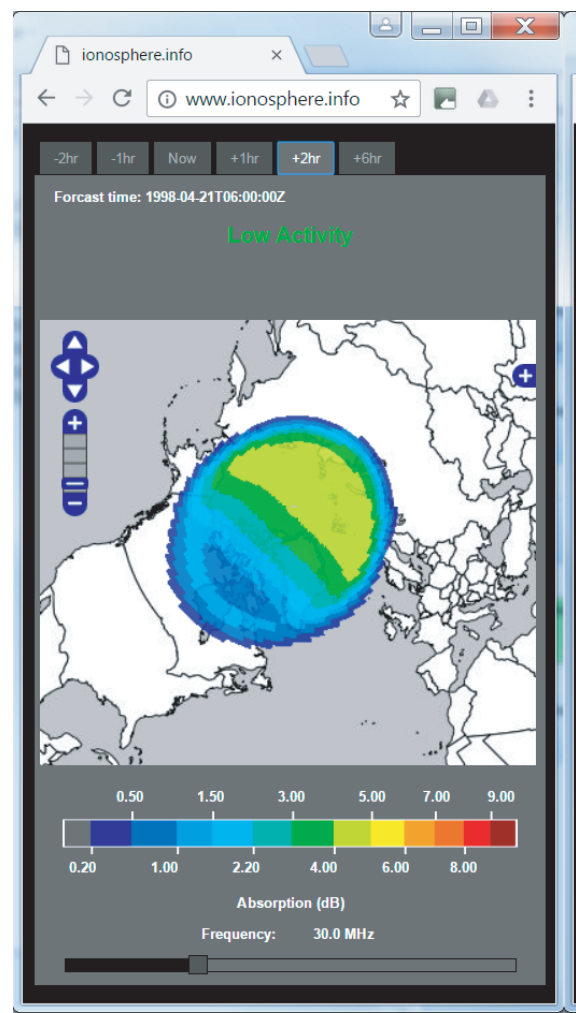

(a)

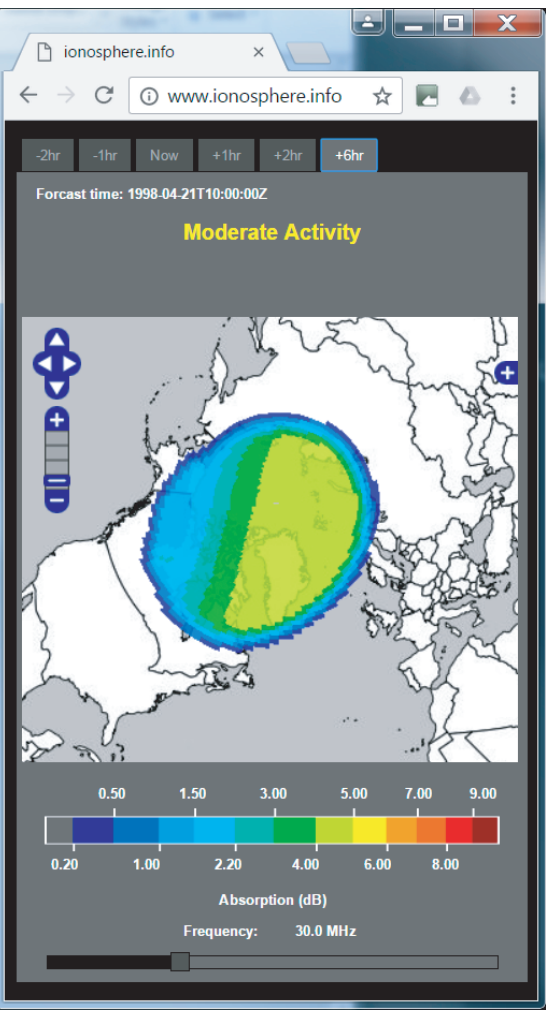

(b)

Figure 6: (b) A prototype web-based interface showing $30 \mathrm{MHz}$ absorption during a Polar Cap Absorption event compared with (a) a period of low activity. 
Based on an analysis from all SPEs in the period 1995-2010, assimilating data from 14 riometers reduced root-mean-square errors by $22-36 \%$ depending on the number of parameters chosen for optimisation [14].

When there are no SPEs or solar flares in progress, HF absorption in the auroral zones is dominated by magnetospheric electron precipitation. This can be localised and sporadic in nature and so is presented as either a probability of $>1 \mathrm{~dB}$ absorption at $30 \mathrm{MHz}$ or as a percentile $\mathrm{dB}$ value, which is determined from an archive of riometer measurements parameterised by geomagnetic indices (or their predictions, based on in-situ solar wind measurements from the DSCOVR satellite [18 and references therein].

A prototype web-based interface has been developed to display predictions of ionospheric absorption over the HF band (see Figure 6). Future development will extend and improve the interface to include signal strength predictions and targetted advice and notices to airline communications services.

\section{CONCLUDING REMARKS}

The aim of this work is to be able to provide an operational service to the airline industry with sufficient information to guide them on whether communications are likely to be maintained on a polar route for the duration of the flight (forecasting) and to provide frequency management information during a flight (nowcasting). Currently the modelling is able to use a range of realtime data sources (e.g., ionosondes, GPS TEC, particle fluxes, etc.) to provide a nowcast of HF ionospheric propagation and absorption conditions that is consistent with measurements made on a number of oblique HF paths. In order to provide forecasts, anticipated space weather conditions need to be incorporated into the model.

\section{ACKNOWLEDGMENT}

The authors are grateful to the EPSRC for their support of this research through grants EP/K008781/1 and EP/K007971/1. This research used the ALICE High Performance Computing Facility at the University of Leicester.

\section{REFERENCES}

1. Warrington, E. M., N. C. Rogers, and T. B. Jones, "Large HF bearing errors for propagation paths contained within the polar cap," IEE Proceedings on Microwaves, Antennas and Propagation, Vol. 144, No. 4, 241-249, 1997.

2. Zaalov, N. Y., E. M. Warrington, and A. J. Stocker, "The simulation of off-great circle HF propagation effects due to the presence of patches and arcs of enhanced electron density within the polar cap ionosphere," Radio Science, Vol. 38, No. 3, 1052, 2003, doi:10.1029/2002RS002798.

3. Rogers, N. C., E. M. Warrington, and T. B. Jones, "Large HF bearing errors for propagationpaths tangential to the auroral oval," IEE Proceedings on Microwaves Antennas and Propagation, Vol. 14, No. 2, 91-96, 1997.

4. Rogers, N. C., E. M. Warrington, and T. B. Jones, "Oblique ionogram features associated with off-great-circle HF propagation at high and sub-auroral latitudes," IEE Proceedings on Microwaves, Antennas and Propagation, Vol. 150, No. 4, 295-300, 2003, doi:10.1049/ipmap:20030552.

5. Siddle, D. R., A. J. Stocker, and E. M. Warrington, "The time-of-flight and direction of arrival of HF radio signals received over a path along the mid-latitude trough: Observations," Radio Science, Vol. 39, RS4008, 2004, doi: 10.1029/2004RS003049.

6. MacDougall, J. and P. T. Jayachandran, "Polar patches: Auroral zone precipitation effects," J. Geophys. Res., Vol. 112, A05312, 2007, doi:10.1029/2006JA011930.

7. Buchau, J., B. W. Reinisch, E. J. Weber, and J. G. Moore, "Structure and dynamics of the winter polar cap F region," Radio Science, Vol. 18, 995-1010, 1983.

8. Jones, R. M. and J. J. Stephenson, A Versatile Three-dimensional Ray Tracing Computer Program for Radio Waves in the Ionosphere, Office of Telecommunications, OT 75-76, U.S Department of Commerce, Washington, USA, 1975.

9. Zaalov, N. Y., E. M. Warrington, and A. J. Stocker, "A ray-tracing model to account for off-great circle HF propagation over northerly paths," Radio Science, Vol. 40, RS4006, 2005, doi: $10.1029 / 2004 R S 003183$. 
10. Bilitza, D., D. Altadill, Y. Zhang, C. Mertens, V. Truhlik, P. Richards, L.-A. McKinnell, and B. Reinisch, "The international reference ionosphere 2012 - A model of international collaboration," Journal of Space Weather and Space Climate, Vol. 4, A07, 2014, doi: $10.1051 / \mathrm{swsc} / 2014004$.

11. Schumer, E. A., "Improved modeling of mid-latitude D-region ionospheric absorption of high frequency radio signals during solar x-ray flares," Ph.D. dissertation, AFIT/DS/ENP/09-J01, United States Air Force, Wright-Patterson Air Force Base, Ohio, USA, June 2009.

12. Smart, D. F., M. A. Shea, E. O. Fluckiger, A. J. Tylka, and P. R. Boberg, "Changes of calculated vertical cutoff rigidities at the altitude of the International Space Station as a function of geomagnetic activity," Proc. Int. Cosmic Ray Conf., Vol. 7, 337-340, 1999.

13. Sauer, H. H. and D. C. Wilkinson, "Global mapping of ionospheric HF/VHF radio wave absorption due to solar energetic protons," Space Weather, Vol. 6, S12002, 2008, doi:10.1029/2008SW000399.

14. Rogers, N. C. and F. Honary, "Assimilation of realtime riometer measurements into models of $30 \mathrm{MHz}$ polar cap absorption," Journal of Space Weather Space Climate, Vol. 5, A8, 2015, doi:10.1051/swsc/2015009.

15. Rogers, N. C., F. Honary, E. M. Warrington, A. J. Stocker, J. Hallam, D. R. Siddle, D. W. Danskin, and B. Jones, "Assimilative real-time models of HF absorption at high latitudes," Proc. 14 th International Ionospheric Effects Symposium (IES 2015), Alexandria, VA, USA, May 1214, 2015.

16. Danskin, D. W., D. Boteler, E. Donovan, and E. Spanswick, "The Canadian riometer array," Proc. 12th International Ionospheric Effects Symposium (IES 2008), 80-86, Alexandria, VA, USA, May 13-15, 2008.

17. Rogers, N. C., A. Kero, F. Honary, P. T. Verronen, and E. M. Warrington, "Improving the twilight model for polar cap absorption nowcasts," Space Weather, Vol. 14, 950-972, 2016, doi: 10.1002/2016SW001527.

18. Wing, S., J. R. Johnson, J. Jen, C.-I. Meng, D. G. Sibeck, K. Bechtold, J. Freeman, K. Costello, M. Balikhin, and K. Takahashi, "Kp forecast models," J. Geophys. Res., Vol. 110, A04203, 2005, doi:10.1029/2004JA010500. 